\title{
On the Max-Min Fair Stochastic Allocation of Indivisible Goods
}

\author{
Yasushi Kawase, ${ }^{1,2}$ Hanna Sumita ${ }^{3}$ \\ ${ }^{1}$ Tokyo Institute of Technology ${ }^{2}$ RIKEN AIP Center \\ ${ }^{3}$ Tokyo Metropolitan University \\ kawase.y.ab@m.titech.ac.jp, sumita@tmu.ac.jp
}

\begin{abstract}
We study the problem of fairly allocating a set of indivisible goods to risk-neutral agents in a stochastic setting. We propose an (approximation) algorithm to find a stochastic allocation that maximizes the minimum utility among the agents. The algorithm runs by repeatedly finding an (approximate) allocation to maximize the total virtual utility of the agents. This implies that the problem is solvable in polynomial time when the utilities are gross-substitutes (which is a subclass of submodular). When the utilities are submodular, we can find a $(1-1 / e)$-approximate solution for the problem and this is best possible unless $\mathrm{P}=\mathrm{NP}$. We also extend the problem where a stochastic allocation must satisfy the (ex ante) envy-freeness. Under this condition, we demonstrate that the problem is NP-hard even when every agent has an additive utility with a matroid constraint (which is a subclass of grosssubstitutes). Furthermore, we propose a polynomial-time algorithm for the setting with a restriction that the matroid constraint is common to all agents.
\end{abstract}

\section{Introduction}

Fair allocation of resources and goods to agents has been a fundamental problem in economic theory over several decades. Recently, a means to find a fair allocation of indivisible goods has received considerable attention in algorithmic game theory, artificial intelligence, and optimization theory (Brandt et al. 2016; Rothe 2016). A motivation is that several practical applications, such as matching courses and allocating jobs to machines, naturally entail fair allocation of indivisible goods. Therefore, there is vast literature addressing many types of settings. In particular, there exist several notions of "fairness"; envy-freeness, max-min fairness, proportionality, max-min share guarantee, etc. exist.

Throughout this paper, we denote the sets of indivisible goods and agents by $E$ and $N$, respectively. Each agent $i \in$ $N$ has a utility function $u_{i}: 2^{E} \rightarrow \mathbb{Z}_{+}$that assigns a value to each set of goods. When each agent $i \in N$ wins a set $X_{i}$ of goods, the utility of agent $i$ is $u_{i}\left(X_{i}\right)$. An allocation is a subpartition of goods. Among notions of fairness, we mainly focus on max-min fairness. A max-min allocation is defined

Copyright (c) 2020, Association for the Advancement of Artificial Intelligence (www.aaai.org). All rights reserved. as one that maximizes the Egalitarian-Social-Welfare (ESW) $\min _{i \in N} u_{i}\left(X_{i}\right)$ (Golovin 2005). This makes the least happy agent as happy as possible.

The goal of this study is to solve the problem of finding a stochastic max-min allocation of indivisible goods. We term this problem MaxESW. A stochastic allocation is a probability distribution on deterministic allocations. In other words, an allocation algorithm uses a lottery to choose a deterministic allocation and distribute the set of goods to each agent. We say that a stochastic allocation satisfies the max-min fairness when the minimum expected utility is maximized. ${ }^{1}$

Regarding motivation, many studies have mentioned that as far as in existing notions, a fair allocation may not exist, or even if exists, there is still room for consideration as to whether it is fair in practice. Consider allocating one good to two agents with positive utilities. As the good is indivisible, either agent can receive it. For max-min allocations, the minimum utility is always zero, and hence any allocation can be fair. However, this is unsatisfactory in practice. Even in this situation, we would like to select either agent in a fair manner.

Stochastic allocation may shed light on the aforementioned difficulty, at least from an ex ante perspective. In the aforementioned case, an algorithm chooses an allocation to either agent with some probability. See also Example 1 in Preliminaries. A lottery can also be interpreted as a fraction of allocation when we repeatedly allocate goods, such as time slots for car sharing during one week. We would like to remark that a stochastic allocation of indivisible goods cannot be naturally interpreted as a deterministic allocation of divisible goods in general. For example, suppose that there are two indivisible goods $E=\{a, b\}$, and one agent with the utility $u(S)=\min \{|S|, 1\}$. The expected utility of allocating $\{a\}$ or $\{b\}$ uniformly at random is 1 , while the expected utility of allocating $\{a, b\}$ or the empty set uniformly at random is $1 / 2$. When we assume that the goods are divisible, natural interpretations of both allocations are the same, namely allocating each good in the ratio of the half. From this fractional allocation, we cannot uniquely determine a corresponding stochastic allocation.

\footnotetext{
${ }^{1}$ The minimum expected utility is not the expectation of the minimum utility.
} 
In this study, we assume the following properties, which are standard in algorithmic game theory and its related areas. (i) No externalities exists, i.e., an agent's utility depends only on the set of goods she wins, not on the identity of the agents that obtain the goods. (ii) Disposal is free (the utility functions are monotone), i.e., $u_{i}(S) \leq u_{i}(T)$ whenever $S \subseteq T$. (iii) The utility functions are normalized, i.e., $u_{i}(\emptyset)=0$ for all agent $i \in N$. (iv) Agents are risk neutral, i.e., the utility of an agent $i$ for a stochastic allocation is naturally defined as the expected utility. (v) We can query the value $u_{i}(S)$ for any agent $i$ and set $S$ of goods.

\section{Related Work}

Many notions of fairness exist, such as envy-freeness (Foley 1967; Varian 1974), proportionality (Steinhaus 1948), and max-min share guarantee (Budish 2011). In particular, envyfreeness is one of the most classical fairness criteria. This guarantees that no agent values any other agent's bundle more than her own bundle. It is known that envy-freeness is a strong condition in a deterministic setting as an envy-free allocation may not exist in general. Therefore, numerous recent work has focused on the relaxations, such as envyfreeness up to one good, denoted by EF1 (Budish 2011), and envy-freeness up to any good, denoted by EFX (Caragiannis et al. 2016).

Finding a deterministic max-min allocation, that maximizes ESW, is quite difficult even when all the utility functions are additive. The additive case is also known as the Santa Clause problem (Bansal and Sviridenko 2006). It is known that this is NP-hard (Bezáková and Dani 2005), and furthermore, it is also NP-hard to approximate it within a factor better than $1 / 2$ (Golovin 2005). Thus, recent work (under additive utility functions) has proposed approximation algorithms. Bansal and Sviridenko (2006) proposed an approximation algorithm for a restricted case. Asadpour and Saberi (2010) provided the first polynomial-time approximation algorithm for the Santa Clause problem, which is improved by Haeupler, Saha, and Srinivasan (2011).

The sum of all utilities, i.e., $\sum_{i \in N} u_{i}\left(X_{i}\right)$, is termed the Utilitarian Social Welfare $(U S W)$. We call the problem of finding a deterministic allocation that maximizes USW MaxUSW. This problem has been extensively studied in the context of combinatorial auction. The optimal allocation can be found in polynomial time when the utilities are gross-substitutes (Bertelsen 2005). Note that any additive function is gross-substitutes, and any gross-substitutes function is submodular. However, when the utilities are budgetadditive, Chakrabarty and Goel (2010) proved that it is NPhard to approximate to a factor better than $15 / 16$. In addition, when the utilities are submodular, Vondrák (2008) provided a $(1-1 / e)$-approximation algorithm (in the value oracle model) and Khot et al. (2008) proved that it is NP-hard to approximate to a factor better than $1-1 / e$ even when all utility functions are the same. For more information, see, e.g., Nisan et al. (2007).

\section{Our Results}

We propose an efficient algorithm for important classes of MaxESW. More precisely, we establish the following results:
1. We prove the NP-hardness of MaxESW even when each utility function is budget-additive (Theorem 2). Moreover, MaxESW is NP-hard to approximate within a factor better than $1-1 / e$, even when all utility functions are the same submodular function (Corollary 1).

2. We propose an $\alpha$-approximation algorithm for MaxESW provided an $\alpha$-approximation algorithm for MaxUSW (Theorem 4). This result enables us to transport existing fruitful results on MaxUSW to MaxESW. In particular, we observe that MaxESW can be solved when every utility function is gross-substitutes, and is $(1-1 / e)$ approximable whenever the utility functions are submodular (Corollaries 2 and 3). This and the aforementioned hardness result imply that our result is tight for the submodular case.

3. We prove the NP-hardness of the problem of finding a stochastic max-min allocation that is also envy-free, even when each utility function is matroid-additive (Theorem 5). We call this problem the envy-free MaxESW.

4. We propose a polynomial-time algorithm for the envyfree MaxESW when all utility functions share the same matroid constraint (Theorem 6).

We will define the aforementioned classes of utility functions in Preliminaries. They are generalizations of additive functions, and often appear in the context of combinatorial auction.

\section{Preliminaries}

A market is a tuple $\left(N, E,\left(u_{i}\right)_{i \in N}\right)$ where each component is defined as follows. There is a finite set $N$ of $n$ risk neutral agents, a finite set $E$ of indivisible goods. Let $u_{i}: 2^{E} \rightarrow \mathbb{Z}_{+}$ denote the utility function of agent $i \in N$. We assume $u_{i}$ is normalized (i.e., $u_{i}(\emptyset)=0$ ) and monotone (i.e., $u(S) \leq$ $u(T)$ for every $S \subseteq T \subseteq E)$. For a set of utility functions $\mathcal{U}$, we call a market $\left(N, \bar{E},\left(u_{i}\right)_{i \in N}\right) \mathcal{U}$-market if $u_{i}$ belongs to $\mathcal{U}$ for every $i \in N$. In this paper, we only consider a set of utility functions that is closed under scaling by a positive factor, i.e., $\lambda \cdot u \in \mathcal{U}$ for any $u \in \mathcal{U}$ and $\lambda>0$.

A list of sets $\boldsymbol{X}=\left(X_{1}, \ldots, X_{n}\right)$ is called allocation if (i) $X_{i} \subseteq E$ for all $i$ and (ii) $X_{i} \cap X_{j}=\emptyset$ for all distinct $i, j \in N$. In other words, an allocation $\boldsymbol{X}=\left(X_{1}, \ldots, X_{n}\right)$ is a subpartition of $E$. When we decide an allocation $\boldsymbol{X}$, an agent $i \in N$ gets $X_{i}$ and her utility is $u_{i}\left(X_{i}\right)$. Let $\mathcal{X}$ be the set of all (feasible ${ }^{2}$ ) allocations. The cardinality of $\mathcal{X}$ is $(|E|+1)^{|N|}$ and hence it is exponential in $|N|$ and $|E|$.

The egalitarian social welfare (ESW) and the utilitarian social welfare (USW) of an allocation $\boldsymbol{X}$ are respectively defined as

$$
\operatorname{ESW}(\boldsymbol{X})=\min _{i \in N} u_{i}\left(X_{i}\right) \text { and } \operatorname{USW}(\boldsymbol{X})=\sum_{i \in N} u_{i}\left(X_{i}\right) .
$$

A max-min allocation is defined as the one that attains $\max _{\boldsymbol{X} \in \mathcal{X}} \operatorname{ESW}(\boldsymbol{X})$ (Golovin 2005).

\footnotetext{
${ }^{2}$ We can handle constraints on allocations (e.g., connectivity constraint (Bouveret et al. 2017; Bilò et al. 2018)) by restricting $\mathcal{X}$.
} 
A stochastic allocation is a probability distribution over $\mathcal{X}$, which is identical to a vector $p \in[0,1]^{\mathcal{X}}$ with $\sum_{\boldsymbol{X} \in \mathcal{X}} p_{\boldsymbol{X}}=1$. We denote by $\Delta(\mathcal{X})$ the set of stochastic allocations. The ESW and USW for a stochastic allocation $p \in \Delta(\mathcal{X})$ are respectively defined as

$$
\begin{aligned}
& \operatorname{ESW}(p)=\min _{i \in N} \mathbb{E}_{\boldsymbol{X} \sim p}\left[u_{i}\left(X_{i}\right)\right]=\min _{i \in N} \sum_{\boldsymbol{X} \in \mathcal{X}} p_{\boldsymbol{X}} u_{i}\left(X_{i}\right), \\
& \operatorname{USW}(p)=\sum_{i \in N} \mathbb{E}_{\boldsymbol{X} \sim p}\left[u_{i}\left(X_{i}\right)\right]=\sum_{i \in N} \sum_{\boldsymbol{X} \in \mathcal{X}} p_{\boldsymbol{X}} u_{i}\left(X_{i}\right) .
\end{aligned}
$$

We say that $p \in \Delta(\mathcal{X})$ is a max-min stochastic allocation if it attains $\max _{p \in \Delta(\mathcal{X})} \operatorname{ESW}(p)$.

We define the problems of maximizing the ESW and the USW, denoted by MaxESW and MaxUSW respectively, as follows. To find a max-min stochastic allocation, we aim to solve MaxESW.

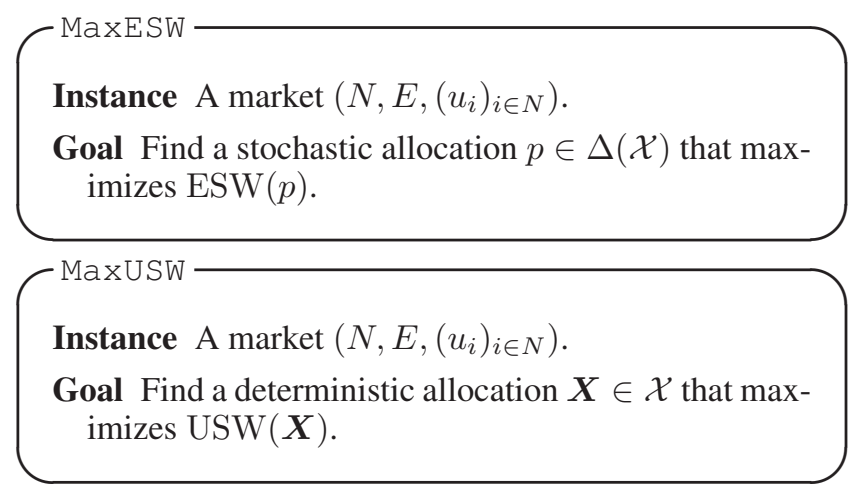

Note that for maximizing $\operatorname{USW}(p)$, there always exists a deterministic optimal one, because $\operatorname{USW}(p)=\sum_{\boldsymbol{X} \in \mathcal{X}} p_{\boldsymbol{X}}$. $\operatorname{USW}(\boldsymbol{X}) \leq \max _{\boldsymbol{X}: p_{\boldsymbol{X}}>0} \operatorname{USW}(\boldsymbol{X})$. Hence, MaxUSW focuses on deterministic allocations. If we restrict the input market to be $\mathcal{U}$-market, we call the problems $\mathcal{U}$-MaxESW and $\mathcal{U}$-MaxUSW.

We briefly mention the size of an output of MaxESW because a stochastic allocation has exponentially many variables. We assume that a stochastic allocation is represented by a sparse form, which is a list containing the index of each non-zero entry together with its value. Let $\operatorname{supp}(p):=$ $\left\{\boldsymbol{X} \in \mathcal{X}: p_{\boldsymbol{X}}>0\right\}$ be the set of non-zero entries of $p$. By Carathéodory's theorem, there exists an optimal stochastic allocation $p$ for $\operatorname{MaxESW}$ such that $|\operatorname{supp}(p)| \leq|N|+1$. $^{3}$ We will find a sparse stochastic allocation, and hence the output of our algorithm has polynomially bounded size.

We also define envy-freeness for stochastic allocations. Given a stochastic allocation $p \in \Delta(\mathcal{X})$, we say that agent $i$ envies agent $j$ if

$$
\mathbb{E}_{\boldsymbol{X} \sim p}\left[u_{i}\left(X_{i}\right)\right]<\mathbb{E}_{\boldsymbol{X} \sim p}\left[u_{i}\left(X_{j}\right)\right]
$$

An allocation without envy is called envy-free (from an ex ante perspective). We will also deal with the problem of find-

\footnotetext{
${ }^{3}$ Carathéodory's theorem states that if a point $x$ in $\mathbb{R}^{n}$ lies in the convex hull of a set $S$, then $x$ can be written as the convex combination of at most $n+1$ points in $S$. In our case, we use the theorem by setting $n=|N|, x=\left(\mathbb{E}_{\boldsymbol{X} \sim p}\left[u_{i}\left(X_{i}\right)\right]\right)_{i \in N}$, and $S=\left\{\left(u_{i}\left(X_{i}\right)\right)_{i \in N}: \boldsymbol{X} \in \mathcal{X}\right\}$.
}

ing a max-min allocation that is also envy-free. The envyfree MaxESW is defined as

$\max \operatorname{ESW}(p) \quad$ s.t. $p \in \Delta(\mathcal{X}), p$ is envy-free.

The next example illustrates difference of optimal deterministic and stochastic allocations.

Example 1. Let us consider a market $\left(N, E,\left(u_{i}\right)_{i \in N}\right)$, where $N=\{1,2\}, E=\{a\}, u_{1}(\{a\})=2, u_{2}(\{a\})=3$. Then, for any deterministic allocation, the ESW is 0 . For a stochastic allocation that chooses an allocation $(\{a\}, \emptyset)$ with probability $3 / 5$ and $(\emptyset,\{a\})$ with probability $2 / 5$, the ESW is $\min \{2 \cdot 3 / 5,3 \cdot 2 / 5\}=6 / 5$.

In addition, $(\emptyset, \emptyset)$ is the unique envy-free deterministic allocation. On the other hand, allocating $\{a\}$ to either agent uniformly at random is envy-free. The ESW of this envyfree stochastic allocation is 1 .

\section{Classes of Utility Functions}

We define classes of utility functions in the following, where each class is closed under scaling by a positive factor. They are well-studied in algorithmic game theory as utilities of agents.

Definition 1. A normalized monotone function $u: 2^{E} \rightarrow$ $\mathbb{R}_{+}$is

- additive, if $u(S)=\sum_{e \in S} u(\{e\})$ for every $S \subseteq E$.

- constrained-additive for a constraint $\mathcal{F} \subseteq 2^{E}$, if $u(S)=$ $\max _{T \subseteq S: T \in \mathcal{F}} \sum_{e \in T} u(\{e\})$ for all $S \subseteq E$. If the constraint is a matroid $^{4}$, we call the function matroidadditive.

- budget-additive, if $u(S)=\min \left\{\sum_{e \in S} u(\{e\}), B\right\}$ for every $S \subseteq E$.

- gross-substitutes, if for every $p, q \in \mathbb{R}^{E}$ with $p \leq q$ and every $S \in \arg \max \left\{u(R)-\sum_{e \in R} p_{v}: R \subseteq E\right\}$, there exists $T \in \arg \max \left\{u(R)-\sum_{e \in R} q_{e}: R \subseteq E\right\}$ such that $\left\{e \in S: p_{e}=q_{e}\right\} \subseteq T$ (Kelso and Crawford 1982).

- submodular, if $u(S)+u(T) \geq u(S \cup T)+u(S \cap T)$ for every $S, T \subseteq E$.

Let us denote by Add, MAdd, BAdd, GS, and SubM the sets of additive functions, of matroid-additive functions, of budget-additive functions, of gross-substitutes functions, and of submodular functions, respectively. It is known that they have the following relationships:

$$
\text { Add } \subsetneq \text { MAdd } \subsetneq \text { GS } \subsetneq \text { SubM and Add } \subsetneq \text { BAdd } \subsetneq \text { SubM }
$$

In addition, some matroid-additive functions are not budgetadditive and some budget-additive functions are not grosssubstitutes.

\footnotetext{
${ }^{4} \mathrm{~A}$ matroid is a pair $(E, \mathcal{F})$ where $\mathcal{F} \subseteq 2^{E}$ with the following properties: (I1) $\emptyset \in \mathcal{F}$, (I2) $X \subseteq Y \in \mathcal{F}$ implies $X \in \mathcal{F}$, and (I3) if $X, Y \in \mathcal{F}$ and $|X|<|Y|$ then there exists $e \in Y \backslash X$ such that $X \cup\{e\} \in \mathcal{F}$. For the basics on matroids, we refer the reader to the book of Oxley (2011).
} 


\section{Identical-agents Case}

In this section, we discuss an easy case in which the utility functions are identical. If the common utility function is additive, an optimal solution is to just allocate each item to an agent uniformly at random. For non-additive utility functions, this strategy does not work. However, we show that a similar strategy works if we can solve MaxUSW.

Let $\left(N=\{1, \ldots, n\}, E,(u)_{i \in N}\right)$ be an arbitrary market on identical agents. For any deterministic allocation $\boldsymbol{X} \in \mathcal{X}$, we define a randomization of $\boldsymbol{X}$ as a stochastic allocation, denoted by $p(\boldsymbol{X})$, such that $(p(\boldsymbol{X}))_{\boldsymbol{X}^{i}}=1 / n$ for each $i \in$ $N$, where $\boldsymbol{X}^{i}=\left(X_{i}, \ldots, X_{n}, X_{1}, \ldots, X_{i-1}\right)$.

Lemma 1. $\operatorname{ESW}(p(\boldsymbol{X}))=\operatorname{USW}(\boldsymbol{X}) / n$ for any $\boldsymbol{X} \in \mathcal{X}$.

Proof. Under the allocation $p(\boldsymbol{X})$, every agent receives $X_{j}$ with a probability $1 / n$ for each $j \in N$, and hence the expected utility of each agent is $\sum_{j \in N} u\left(X_{j}\right) / n$. Thus, $\operatorname{ESW}(p(\boldsymbol{X}))=\min _{i \in N} \sum_{j \in N} u\left(X_{j}\right) / n=\mathrm{USW}(\boldsymbol{X}) / n$.

Theorem 1. For any market $\left(N, E,(u)_{i \in N}\right)$ on identical agents and any positive real $\alpha \leq 1$, an $\alpha$-approximate solution for MaxESW can be constructed in polynomial time from an $\alpha$-approximate solution for MaxUSW, and vice versa.

Proof. Suppose that $\boldsymbol{X}^{\prime} \in \mathcal{X}$ is an $\alpha$-approximate solution for MaxUSW, i.e., $\operatorname{USW}\left(\boldsymbol{X}^{\prime}\right) \geq \alpha \cdot \max _{\boldsymbol{X} \in \mathcal{X}} \operatorname{USW}(\boldsymbol{X})$. Let $p^{*}$ be the optimal solution for MaxESW. We take arbitrarily $\boldsymbol{X}^{*} \in \arg \max _{\boldsymbol{X} \in \operatorname{supp}\left(p^{*}\right)} \operatorname{USW}(\boldsymbol{X})$. Then, by Lemma 1,

$$
\begin{aligned}
\operatorname{ESW}\left(p\left(\boldsymbol{X}^{\prime}\right)\right) & =\operatorname{USW}\left(\boldsymbol{X}^{\prime}\right) / n \geq \alpha \cdot \max _{\boldsymbol{X} \in \mathcal{X}} \operatorname{USW}(\boldsymbol{X}) / n \\
& \geq \alpha \cdot \operatorname{USW}\left(\boldsymbol{X}^{*}\right) / n \geq \alpha \cdot \operatorname{USW}\left(p^{*}\right) / n \\
& =\alpha \cdot \sum_{j \in N}(1 / n) \sum_{\boldsymbol{X} \in \operatorname{supp}\left(p^{*}\right)} p_{\boldsymbol{X}} u\left(X_{j}\right) \\
& \geq \alpha \cdot \operatorname{ESW}\left(p^{*}\right) .
\end{aligned}
$$

Hence, $p\left(\boldsymbol{X}^{\prime}\right)$ is an $\alpha$-approximate solution for MaxESW.

Conversely, suppose that $p^{\prime}$ is an $\alpha$-approximate solution for MaxESW, i.e., $\operatorname{ESW}\left(p^{\prime}\right) \geq \alpha$. $\max _{p \in \Delta(\mathcal{X})} \operatorname{ESW}(p)$. Let $\boldsymbol{X}^{\prime}$ be an allocation such that $\boldsymbol{X}^{\prime} \in \arg \max _{\boldsymbol{X} \in \operatorname{supp}\left(p^{\prime}\right)} \operatorname{USW}(\boldsymbol{X})$. Let $\boldsymbol{X}^{*}$ be an optimal solution for MaxUSW. Then, by Lemma 1,

$$
\begin{aligned}
\operatorname{USW}\left(\boldsymbol{X}^{\prime}\right) & \geq \operatorname{USW}\left(p^{\prime}\right) \geq n \cdot \operatorname{ESW}\left(p^{\prime}\right) \\
& \geq \alpha n \cdot \max _{p \in \Delta(\mathcal{X})} \operatorname{ESW}(p) \\
& \geq \alpha n \cdot \operatorname{ESW}\left(p\left(\boldsymbol{X}^{*}\right)\right)=\alpha \cdot \operatorname{USW}\left(\boldsymbol{X}^{*}\right) .
\end{aligned}
$$

Hence, $\boldsymbol{X}^{\prime}$ is an $\alpha$-approximate solution for MaxUSW.

It should be noted that the stochastic allocation $p\left(\boldsymbol{X}^{\prime}\right)$ in the aforementioned proof is envy-free by the symmetry of the agents. Hence, the optimal value of MaxESW is equal to that of the envy-free MaxESW.

Khot et al. (2008) proved that MaxUSW is NP-hard to approximate within a factor better than $1-1 / e$ even when all utility functions are the same submodular function. Therefore, by Theorem 1, we have the following Corollary.
Corollary 1. MaxESW and the envy-free MaxESW are NPhard to approximate within a factor better than $1-1 / e$, even when all utility functions are the same submodular function.

Finally, we show NP-hardness of a restricted MaxESW problem by reducing the partition problem, which is known to be NP-hard (Garey and Johnson 1979).

Partition problem

Instance $\mathrm{A}$ list of $m$ positive integers $a_{1}, \ldots, a_{m}$.

Question Is there a subset $A \subseteq\{1, \ldots, m\}$ such that

$$
\sum_{j \in A} a_{j}=\sum_{j \notin \backslash A} a_{j} \text { ? }
$$

Theorem 2. MaxESW and the envy-free MaxESW are NPhard, even when there are only two agents with the same budget-additive utility function.

Proof. Let $\left(a_{1}, \ldots, a_{m}\right)$ be an instance of the partition problem. We may assume that $\sum_{j=1}^{m} a_{j}$ is even because otherwise, the answer is No. Let $M=\sum_{j=1}^{m} a_{j} / 2$. We construct a market $\left(N:=\{1,2\}, E,(u)_{i \in N}\right)$, where $E=\{1, \ldots, m\}$ and $u: 2^{E} \rightarrow \mathbb{Z}_{+}$satisfies $u(X)=\min \left\{\sum_{j \in X} a_{j}, M\right\}$. Then, the optimal value is at least $M$ if and only if the given partition problem instance is a Yes-instance. Indeed, if there is $A^{*} \subseteq E$ such that $\sum_{j \in A^{*}} a_{j}=\sum_{j \in E \backslash A^{*}} a_{j}(=M)$, then $\boldsymbol{X}=\left(A^{*}, E \backslash A^{*}\right)$ satisfies $\operatorname{ESW}(\boldsymbol{X}) \geq M$ and it is envy-free. Also, assume that there is $p^{*} \in \Delta(\mathcal{X})$ such that $\operatorname{ESW}\left(p^{*}\right) \geq M$. For some $\boldsymbol{X}^{*} \in \operatorname{supp}\left(p^{*}\right)$, we have $\operatorname{ESW}\left(\boldsymbol{X}^{*}\right) \geq \bar{M}$, and hence $M \leq u\left(X_{1}^{*}\right) \leq \sum_{j \in X_{1}^{*}} a_{j}$ and $M \leq u\left(X_{2}^{*}\right) \leq u\left(E \backslash X_{1}^{*}\right) \leq \sum_{j \in E \backslash X_{1}^{*}} a_{j}$. Therefore, $\sum_{j \in X_{1}^{*}} a_{j}=\sum_{j \in E \backslash X_{1}^{*}} a_{j}=M$.

\section{Dual-based algorithm}

In this section, we propose an $\alpha$-approximation algorithm for MaxESW given an $\alpha$-approximation algorithm for MaxUSW. Let $\left(N, E,\left(u_{i}\right)_{i \in N}\right)$ be a $\mathcal{U}$-market, where the utility functions are not necessarily identical. Without loss of generality, we assume that the optimal value of MaxESW is positive (this assumption holds if and only if $u_{i}(E)>0$ for all $i \in N$, and hence we can easily check it). Then, MaxESW for $\left(N, E,\left(u_{i}\right)_{i \in N}\right)$ is formulated as follows:

$$
\begin{array}{rlr}
\max & t & \\
\text { s.t. } & t \leq \sum_{\boldsymbol{X} \in \mathcal{X}} u_{i}\left(X_{i}\right) p_{\boldsymbol{X}} & (\forall i \in N), \\
& \sum_{\boldsymbol{X} \in \mathcal{X}} p_{\boldsymbol{X}}=1, & (\forall \boldsymbol{X} \in \mathcal{X}), \\
& p_{\boldsymbol{X} \geq 0} \geq 0 .
\end{array}
$$

By setting $q_{\boldsymbol{X}}:=p_{\boldsymbol{X}} / t(\boldsymbol{X} \in \mathcal{X})$, we transform (3) to an exponentially-large Linear Programming problem (LP) whose optimal value is the inverse of the one for (3) as follows:

$$
\begin{array}{cll}
\min & \sum_{\boldsymbol{X} \in \mathcal{X}} q_{\boldsymbol{X}} & \\
\text { s.t. } & \sum_{\boldsymbol{X} \in \mathcal{X}} u_{i}\left(X_{i}\right) q_{\boldsymbol{X}} \geq 1 \quad(\forall i \in N), \\
& q_{\boldsymbol{X}} \geq 0 & (\forall \boldsymbol{X} \in \mathcal{X}) .
\end{array}
$$


The dual is given by:

$$
\begin{array}{rll}
\max & \sum_{i \in N} z_{i} & \\
\text { s.t. } & \sum_{i \in N} u_{i}\left(X_{i}\right) z_{i} \leq 1 & (\forall \boldsymbol{X} \in \mathcal{X}), \\
& z_{i} \geq 0 & (\forall i \in N) .
\end{array}
$$

In the following, we aim to solve (4) via solving (5) by the ellipsoid algorithm. This algorithm works when we have a separation algorithm to solve the separation problem (Grötschel, Lovász, and Schrijver 2012). For a polyhedron $P \subseteq \mathbb{R}^{n}$, the separation problem for $P$ receives a vector $y$ and either asserts $y \in P$ or finds a vector $d$ such that $d^{\top} x>d^{\top} y$ for all $x \in P$. We need a separation algorithm for the feasible region on (5). Fortunately, in our case, checking the feasibility of a given input $\hat{z} \in \mathbb{R}^{N}$ is equivalent to solving MaxUSW for $\left(N, E,\left(\hat{z}_{i} u_{i}\right)_{i \in N}\right)$. If $\hat{z}_{i}<0$ for some $i \in N$, then $\hat{z}$ is infeasible and $-\hat{z}_{i}>0 \geq-z_{i}$ for all feasible solutions $z \in \mathbb{R}^{N}$. Assume that $\hat{z}_{i} \geq 0$ for all $i \in N$. If the optimal solution $\boldsymbol{X}^{*}$ of MaxUSW for $\left(N, E,\left(\hat{z}_{i} u_{i}\right)_{i \in N}\right)$ has the objective value larger than $1,\left(u_{i}\left(X_{i}^{*}\right)\right)_{i \in N}$ is a desired vector as $d$, because $\sum_{i \in N} u_{i}\left(X_{i}^{*}\right) \hat{z}_{i}>1 \geq \sum_{i \in N} u_{i}\left(X_{i}^{*}\right) z_{i}$ for all feasible solutions $z \in \mathbb{R}^{N}$. Note that $\left(N, E,\left(\hat{z}_{i} u_{i}\right)_{i \in N}\right)$ is a $\mathcal{U}$-market. When every utility function is gross-substitutes, MaxUSW is polynomially solvable (Bertelsen 2005), and so is the separation problem. Therefore, MaxESW with gross-substitute utility functions is solvable in polynomial time. However, we cannot apply the method when the utility functions are submodular or more general.

As a remedy, we employ the technique of Jansen (2003) that uses an approximate separation algorithm to solve the LP approximately. For a polyhedron $P \subseteq \mathbb{R}^{n}$ and a positive $\alpha \leq 1$, the $\alpha$-approximate separation algorithm for $P$ receives a vector $y$ and either asserts $\alpha y \in P$ or finds a vector $d$ such that $d^{\top} x>d^{\top} y$ for all $x \in P$. Jansen (2003) focused on the LP of the form:

$$
\begin{aligned}
\max & c^{\top} y \\
\text { s.t. } & a_{j}^{\top} y \leq b_{j} \quad(\forall j=1, \ldots, m), \\
& y \in B,
\end{aligned}
$$

where $a_{j}$ is an $n$-dimensional rational vector and $b_{j}$ is a positive rational number for all $j$, and $B$ is a polytope defined by some inequalities such that the separation problem for $B$ is solvable in polynomial time. Let $K$ be the feasible region of (6). Then, Jansen (2003) showed the following result. Suppose that there is an $\alpha$-approximation algorithm APP that finds a row $a_{\ell}^{\top} y \leq b_{\ell}$ with

$$
a_{\ell}^{\top} y / b_{\ell} \geq \alpha \cdot \max _{j \in\{1, \ldots, m\}} a_{j}^{\top} y / b_{j}
$$

for each $y \in B$, and we know an integer $\psi$ such that all the rational inequalities defining $K$ are of size at most $\psi$. Then, there is a polynomial-time $\alpha$-approximate separation algorithm for (6). Moreover, there is a $1 / \alpha$-approximation algorithm for the dual of (6) that runs in polynomial time in $n, \psi$ and the running time of APP.

We utilize the result of Jansen (2003) as follows. We set $c$ to be the all-one vector and $B=\left\{z: z_{i} \geq 0(\forall i \in\right.$ $N)\}$. Let $\mu=\max _{i \in N} u_{i}(E)$. We set $\psi=\bar{O}(n \log \mu)$. Indeed, each constraint in (5) has at most $n$ coefficients of size bounded by $\log \left(\max _{i \in N, \boldsymbol{X} \in \mathcal{X}} u_{i}\left(X_{i}\right)\right) \leq \log \mu$ because each $u_{i}$ is monotone. In addition, we use an $\alpha$ approximation algorithm APP for the $\mathcal{U}$-MaxUSW: Given $\left(N, E,\left(u_{i}^{\prime}\right)_{i \in N}\right)$ with $u_{i}^{\prime} \in \mathcal{U}(\forall i \in N)$, the algorithm APP finds an allocation $\tilde{\boldsymbol{X}} \in \mathcal{X}$ such that $\sum_{i \in N} u_{i}^{\prime}\left(\tilde{X}_{i}\right) \geq$ $\alpha \cdot \max _{\boldsymbol{X} \in \mathcal{X}} \sum_{i \in N} u_{i}^{\prime}\left(X_{i}\right)$ in polynomial time with respect to $n, \log \mu$, and the time to evaluate the value of $u_{i}^{\prime}(i \in N)$.

According to Jansen (2003), we define an approximate separation algorithm that receives $z$, as Algorithm 1 .

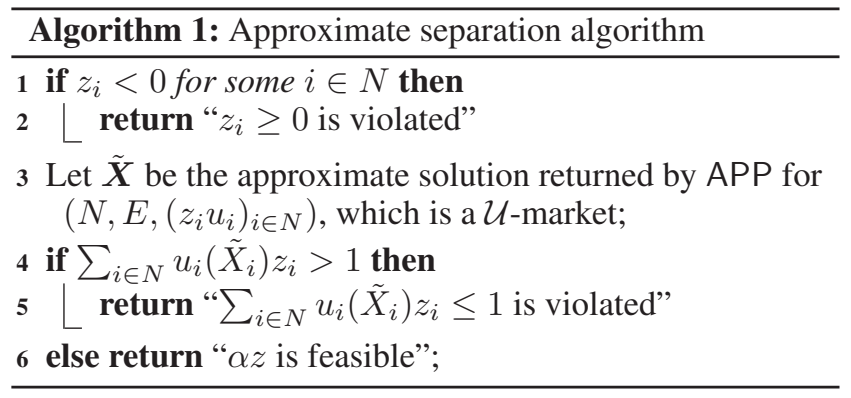

We can see that Algorithm 1 returns an answer in polynomial time in $n$ and the running time of the value oracle of $u_{i}(i \in N)$. When it says that $\alpha z$ is feasible, this is correct because $1 \geq \sum_{i \in N} u_{i}\left(\tilde{X}_{i}\right) z_{i} \geq \alpha \cdot \sum_{i \in N} u_{i}\left(X_{i}\right) z_{i}$ for all $\boldsymbol{X} \in \mathcal{X}$ by the construction.

Then, we solve (4) by Algorithm 2.

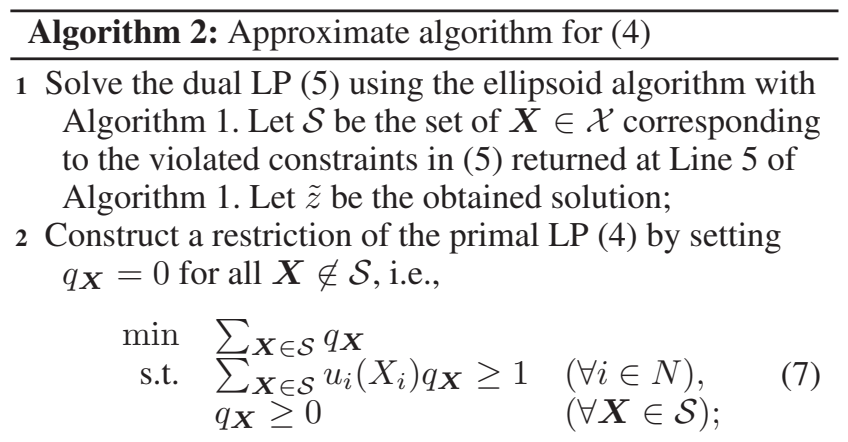

3 Solve LP (7) with a standard LP algorithm (such as the algorithm by Khachiyan (1980));

4 return an optimal solution $\left(\tilde{q}_{\boldsymbol{X}}\right)_{\boldsymbol{X} \in \mathcal{S}}$ of LP (7);

We remark that $\mathcal{S}$ has polynomial size. The size of $\mathcal{S}$ increases by at most one per one call for Algorithm 1. Moreover, by construction of the ellipsoid algorithm, the number of the calls for Algorithm 1 is bounded by a polynomial (in $n, \log \mu$, and time to evaluate $u_{i}$ 's).

We can rewrite the result of Jansen (2003) as follows.

Theorem 3 (Jansen (2003)). Suppose that there is an $\alpha$ approximation algorithm APP for the $\mathcal{U}$-MaxUSW. Then, Algorithm 1 is a polynomial-time $\alpha$-approximate separation algorithm for (5), and Algorithm 2 is a $1 / \alpha$-approximation algorithm for (4) that runs in polynomial time in $n, \log \mu$ and the running time of APP. 
From $\left(\tilde{q}_{\boldsymbol{X}}\right)_{\boldsymbol{X} \in \mathcal{S}}$, we construct $\tilde{t}=1 / \sum_{\boldsymbol{X} \in \mathcal{S}} \tilde{q}_{\boldsymbol{X}}$ and $\tilde{p}_{\boldsymbol{X}}=\tilde{t} \cdot \tilde{q}_{\boldsymbol{X}}$ for $\boldsymbol{X} \in \mathcal{S}$. We provide a theoretical guarantee on this solution for (3).

Theorem 4. With an $\alpha$-approximation algorithm APP for the $\mathcal{U}$-MaxUSW, we can obtain an $\alpha$-approximation algorithm for the $\mathcal{U}$-MaxESW in polynomial time with respect to $n, \log \mu$, and the time to evaluate the value of $u_{i}(i \in N)$.

Proof. We see from Theorem 3 that Algorithm 2 terminates in polynomial time. The construction of $\left(\left(\tilde{p}_{\boldsymbol{X}}\right)_{\boldsymbol{X} \in \mathcal{S}}, \tilde{t}\right)$ can be done also in polynomial time. Since $\left(\tilde{q}_{\boldsymbol{X}}\right)_{\boldsymbol{X} \in \mathcal{S}}$ is feasible for (4), $\left(\left(\tilde{p}_{\boldsymbol{X}}\right)_{\boldsymbol{X} \in \mathcal{S}}, \tilde{t}\right)$ is feasible for (3). Let OPT be the optimal value of (3). By construction, the optimal value of (4) is equal to $1 / \mathrm{OPT}$. By Theorem 3, we have $\sum_{\boldsymbol{X} \in \mathcal{S}} \tilde{q}_{\boldsymbol{X}} \leq(1 / \alpha) \cdot(1 / \mathrm{OPT})$. Thus, it holds that $\alpha \cdot$ OPT $\leq 1 / \sum_{\boldsymbol{X} \in \mathcal{S}} \tilde{q}_{\boldsymbol{X}}=\tilde{t}$. Therefore, $\left(\left(\tilde{p}_{\boldsymbol{X}}\right)_{\boldsymbol{X} \in \mathcal{S}}, \tilde{t}\right)$ is an $\alpha$-approximate solution for (3).

We apply existing results on MaxUSW to Theorem 4. If $\mathcal{U}$ is the set GS of gross-substitutes functions, then it is known that the $\mathcal{U}$-MaxUSW is solvable in polynomial time (Bertelsen 2005).

Corollary 2. There exists a polynomial-time algorithm for the GS-MaxESW.

If $\mathcal{U}$ is the set SubM of submodular functions, then there exists a $(1-1 / e)$-approximation algorithm for the $\mathcal{U}$ MaxUSW (Vondrák 2008).

Corollary 3. There exists a $(1-1 / e)$-approximation algorithm for the SubM-MaxESW.

Due to Corollary 1, this is best-possible unless $\mathrm{P}=\mathrm{NP}$.

\section{Envy-free Max-min Allocation}

In this section, we focus on the envy-free MaxESW (2). We remark that allocating the entire bundle of goods to an agent chosen uniformly at random is an $n$-approximation solution since it is envy-free and $\min _{i \in N} u_{i}(E) \geq$ $\max _{p \in \Delta(\mathcal{X})} \operatorname{ESW}(p)$. We first prove that the envy-free version is NP-hard even if each utility is matroid-additive. However, we also show that the problem is polynomial-time solvable if all constraints are the same matroid. This assumption is critical for polynomial-time solvability under general matroids, due to the hardness result.

Before going to the main part, we mention an interesting property of the envy-free MaxESW. It seems intuitive that when a utility function of some agent is replaced with $u_{i}^{\prime}$ such that $u_{i}^{\prime}(S) \geq u_{i}(S)$ for all $S$, the ESW will become higher. This is true for MaxESW because the optimal allocation for the original market is also feasible for the increased market. However, it is not the case for the envy-free MaxESW.

Example 2. Let us consider a market $(N:=\{1,2\}, E:=$ $\left.\{a, b, c\},\left(u_{1}, u_{2}\right)\right)$ where $u_{1}(X)=\min \{2|X|, 2\}$ and $u_{2}(X)=|X|$ for $X \subseteq E$. Then, an optimal solution to the envy-free MaxESW is an allocation that chooses $(\{a\},\{b, c\})$ with probability 1 , and the optimal ESW value is 2. Meanwhile, under a market $\left(N, E,\left(u_{1}^{\prime}, u_{2}\right)\right)$ with $u_{1}^{\prime}(X)=2|X|$, an optimal solution is to distribute the goods independently and uniformly at random, and the optimal ESW value is 1.5 .

\section{NP-hardness for General Constraints}

We prove that the envy-free MaxESW is NP-hard, even when the utility function $u_{i}$ of each agent $i \in N$ is matroidadditive with a matroid $\left(E, \mathcal{F}_{i}\right)$.

We give a reduction from the following Partition into Triangles problem (PT), which is NP-complete (Garey and Johnson 1979).

Partition into Triangles (PT)

Instance A graph $G=(V, E)$, with $|V|=3 q$ for some integer $q$.

Question Can the vertices $V$ be partitioned into $q$ disjoint sets $V_{1}, V_{2}, \ldots, V_{q}$, each containing exactly 3 vertices, such that for each of these $V_{i}$ is the vertex set of a triangle in $G$ (i.e., $\left\{v_{i}^{1}, v_{i}^{2}\right\},\left\{v_{i}^{1}, v_{i}^{3}\right\},\left\{v_{i}^{2}, v_{i}^{3}\right\} \in E$ for all $V_{i}=$ $\left.\left\{v_{i}^{1}, v_{i}^{2}, v_{i}^{3}\right\}(i=1, \ldots, q)\right)$ ?

Without loss of generality, we may assume that $|E| \geq 3 q$, since otherwise the instance must be a No-instance.

We construct a market $\left(N, R,\left(u_{i}\right)_{i \in N}\right)$ that has an envyfree stochastic allocation with ESW at least 6 if and only if the given PT instance is a Yes-instance. The overview of the market is illustrated in Figure 1. Formally, the agents and goods are set as

- $N=T \cup S \cup\{a\}$, where $T:=\left\{t_{1}, \ldots, t_{q}\right\}$ and $S:=$ $\left\{s_{1}, \ldots, s_{|E|-3 q}\right\}$,

- $R=V \cup E \cup\{\ell\}$.

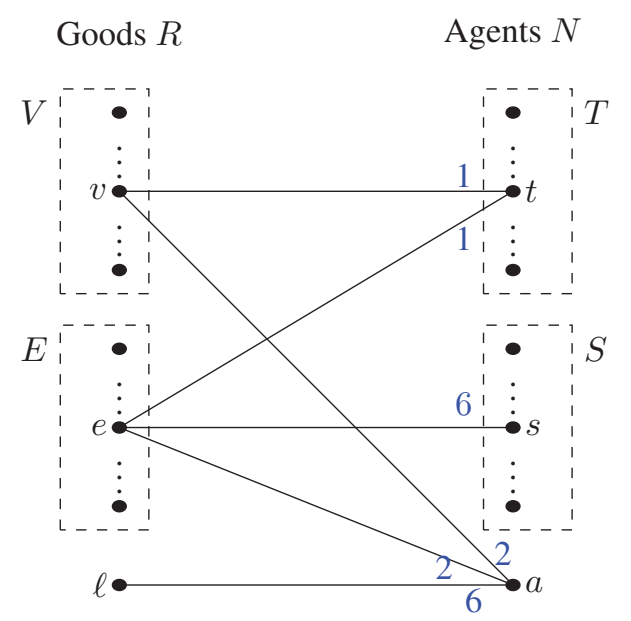

Figure 1: The construction of a market from a given $\mathrm{PT}$ instance.

We define the utility functions for all agents as the following constrained-additive ones with matroids.

- For $u_{t}(t \in T)$,

- $\mathcal{F}_{t}=\{X \subseteq V \cup E:|X \cap V| \leq 3,|X \cap E| \leq 3\}$, 
- $u_{t}(\{r\})=1(\forall r \in R)$;

- For $u_{s}(s \in S)$,

- $\mathcal{F}_{s}=\{X \subseteq E:|X| \leq 1\}$,

- $u_{s}(\{r\})=6(\forall r \in R)$;

- For $u_{a}$,

- $u_{a}(\{\ell\})=6, u_{a}(\{r\})=2(\forall r \in V \cup E)$,

- $\mathcal{F}_{a}$ forms a binary matroid represented by vectors $\chi_{\ell}$, $\chi_{v}(v \in V)$, and $\chi_{e}(e \in E)$ in $\mathbb{F}_{2}^{\{a\} \cup V}$, where

$* \chi_{\ell, a}=1, \chi_{\ell, v}=0(v \in V)$,

* $\chi_{v, v}=1, \chi_{v, w}=0(w \neq v)$,

$* \chi_{\{v, w\}, v}=\chi_{\{v, w\}, w}=1, \chi_{\{v, w\}, x}(x \neq v, w)$.

A set $X \subseteq R$ is in $\mathcal{F}_{a}$ if the corresponding vectors are linearly independent in $\mathbb{F}_{2}$.

These vectors form a matrix as follows:

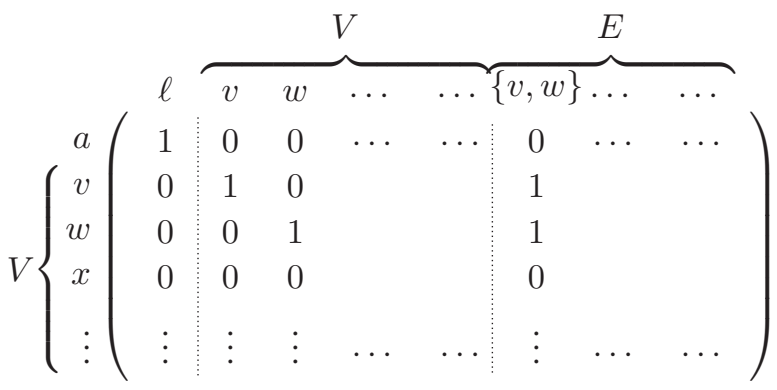

Intuitively, to attain ESW at least 6, each $t \in T$ must be allocated 3 goods of $V$ and of $E$, respectively. In addition, each $s \in S$ must be allocated one good of $R_{E}$.

Suppose that the PT instance is a Yes-instance, i.e., there exists a partition $V_{1}^{*} \ldots, V_{q}^{*}$ of $V$ such that each $V_{i}^{*}(i \in\{1, \ldots, q\})$ is a vertex set of a triangle in $G$. For $i=1, \ldots, q$, let $V_{i}^{*}=\left\{v_{i}^{1}, v_{i}^{2}, v_{i}^{3}\right\}$, $E_{i}^{*}=\left\{\left\{v_{i}^{1}, v_{i}^{2}\right\},\left\{v_{i}^{1}, v_{i}^{3}\right\},\left\{v_{i}^{2}, v_{i}^{3}\right\}\right\}$, and $E \backslash \bigcup_{i=1}^{q} E_{i}^{*}=$ $\left\{e_{1}, \ldots, e_{|E|-3 q}\right\}$. Let $\boldsymbol{X}^{*} \in \mathcal{X}$ be a deterministic allocation defined as $X_{a}^{*}=\{\ell\}, X_{t_{i}}^{*}=V_{i}^{*} \cup E_{i}^{*}(i \in\{1, \ldots, q\})$, and $X_{s_{i}}^{*}=\left\{e_{i}\right\}(i \in\{1, \ldots,|E|-3 q\})$. We see that each agent receives a feasible goods set. Every agent has utility 6 , and hence $\operatorname{ESW}\left(\boldsymbol{X}^{*}\right)=6$. We claim that $\boldsymbol{X}^{*}$ is envy-free.

- For every $t \in T, u_{t}\left(X_{t^{\prime}}\right)=6\left(\forall t^{\prime} \in T\right)$ (because $X_{t^{\prime}} \in$ $\left.\mathcal{F}_{t}\right), u_{t}\left(X_{s}\right)=1(\forall s \in S)$ and $u_{t}\left(X_{a}\right)=1$.

- For $s \in S, u_{s}\left(X_{t}\right)=6(t \in T), u_{s}\left(X_{s^{\prime}}\right)=6\left(\forall s^{\prime} \in S\right)$ and $u_{s}\left(X_{a}\right)=0$.

- For $a$, we see that $u_{a}\left(X_{t}\right)=6(\forall t \in T)$ because the rank of $X_{t}$ is 3 , and $u_{a}\left(X_{s}\right)=2(\forall s \in S)$.

Thus, the instance has an envy-free (stochastic) allocation with ESW at least 6 .

Conversely, suppose that the $\mathrm{P} \mathrm{T}$ instance is a No-instance, i.e., there exists no partition into triangles. Suppose that $p^{*} \in$ $\Delta(\mathcal{X})$ is a stochastic allocation such that $\operatorname{ESW}\left(p^{*}\right) \geq 6$. We claim that $p^{*}$ is not envy-free. For any deterministic feasible allocation $\boldsymbol{X} \in \mathcal{X}, \operatorname{ESW}(\boldsymbol{X}) \leq 6$ because $u_{i}\left(X_{i}\right) \leq 6$ for any agent $i \in T \cup S$. Then, $\operatorname{ESW}\left(p^{*}\right) \leq 6$, and hence $\operatorname{ESW}\left(p^{*}\right)=6$. Thus, we have $\operatorname{ESW}(\boldsymbol{X})=6$ for any $\boldsymbol{X} \in$ $\operatorname{supp}\left(p^{*}\right)$. Fix an deterministic allocation $\hat{\boldsymbol{X}} \in \operatorname{supp}\left(p^{*}\right)$. Since $u_{i}\left(\hat{X}_{i}\right) \geq 6$ for all agents $i$, we have $u_{i}\left(\hat{X}_{i}\right)=6$ for any $i \in T \cup S$. This is attained only if $\left|\hat{X}_{t} \cap V\right|=3$, $\left|\hat{X}_{t} \cap E\right|=3(t \in T)$, and $\left|\hat{X}_{s} \cap E\right|=1(s \in S)$ by construction. In this case, all goods in $V \cup E$ are allocated to agents in $T \cup S$. To attain $u_{a}\left(\hat{X}_{a}\right) \geq 6$, we have $\hat{X}_{a}=\{\ell\}$. Hence,

$$
u_{a}\left(\hat{X}_{a}\right)=6 \quad \text { and } \quad u_{a}\left(\hat{X}_{i}\right) \geq 6(\forall i \in T) .
$$

Note that this holds for all $\boldsymbol{X} \in \operatorname{supp}\left(p^{*}\right)$ by the same argument. As the PT instance is a No-instance, there exists $t^{*} \in T$ such that $\hat{X}_{t^{*}}$ does not form a triangle, i.e., there exists $\left\{w, w^{\prime}\right\} \in \hat{X}_{t^{*}} \cap E$ such that $w^{\prime} \notin \hat{X}_{t^{*}} \cap V$. Thus, the four vectors $\chi_{v}\left(v \in \hat{X}_{t^{*}} \cap V\right)$ and $\chi_{\left\{w, w^{\prime}\right\}}$ are independent for $\mathcal{F}_{a}$. Hence, $u_{a}\left(\hat{X}_{t^{*}}\right)$ is at least $2 \times 4=8$. Therefore, this together with (8) implies that

$$
\begin{gathered}
\sum_{\boldsymbol{X} \in \mathcal{X}} p_{\boldsymbol{X}^{*}}^{*} u_{a}\left(X_{t^{*}}\right)=p_{\hat{\boldsymbol{X}}}^{*} u_{a}\left(\hat{X}_{t^{*}}\right)+\sum_{\boldsymbol{X} \in \mathcal{X} \backslash\{\hat{\boldsymbol{X}}\}} p_{\boldsymbol{X}^{*}}^{*} u_{a}\left(X_{t^{*}}\right) \\
\geq p_{\hat{\boldsymbol{X}}}^{*} \cdot 8+\left(1-p_{\hat{\boldsymbol{X}}}^{*}\right) \cdot 6>6=\sum_{\boldsymbol{X} \in \mathcal{X}} p_{\boldsymbol{X}}^{*} u_{a}\left(X_{a}\right),
\end{gathered}
$$

which means that $p^{*}$ is not envy-free. This completes the proof of the following theorem.

Theorem 5. The envy-free MaxESW problem is NP-hard even when the utility functions are matroid-additive.

\section{Polynomial-time Algorithm for Identical Constraints}

In this subsection, we show that we can solve the envy-free MaxESW in polynomial time if all agents have the same matroid, i.e., $\mathcal{F}_{i}=\mathcal{F}(\forall i \in N)$.

Theorem 6. If all utility functions are matroid-additive with a common matroid, then the envy-free MaxESW is solvable in polynomial time.

Let us focus on the exponentially-large LP representation of the envy-free MaxESW:

$$
\begin{array}{rlr}
\max & t & \\
\text { s.t. } & t \leq \sum_{\boldsymbol{X} \in \mathcal{X}} u_{i}\left(X_{i}\right) p_{\boldsymbol{X}} & (\forall i \in N), \\
& \sum_{\boldsymbol{X} \in \mathcal{X}}\left(u_{i}\left(X_{i}\right)-u_{i}\left(X_{j}\right)\right) p_{\boldsymbol{X}} \geq 0 & (\forall i, j \in N), \\
& \sum_{\boldsymbol{X} \in \mathcal{X}} p_{\boldsymbol{X}}=1, & (\forall \boldsymbol{X} \in \mathcal{X}) .
\end{array}
$$

Its dual is given by:

$$
\begin{aligned}
& \begin{aligned}
\min & s \\
\text { s.t. } & s \geq \sum_{i \in N} u_{i}\left(X_{i}\right) z_{i}
\end{aligned} \\
& +\sum_{i, j \in N}\left(u_{i}\left(X_{i}\right)-u_{i}\left(X_{j}\right)\right) y_{i j} \quad(\forall \boldsymbol{X} \in \mathcal{X}), \\
& \sum_{i \in N} z_{i}=1 \text {, } \\
& y_{i j} \geq 0, z_{i} \geq 0 \\
& (\forall i, j \in N) \text {. }
\end{aligned}
$$

In a similar way to the algorithm presented in the previous section, if we can solve the separation problem for the dual LP, then we can compute the optimal solution to the envyfree MaxESW. Thus, we show the polynomial-time solvability of the separation problem for the dual LP.

Let $(z, y, s)$ be a given input. If (i) $y_{i j}<0$ for some $i, j$, (ii) $\sum_{i \in N} z_{i} \neq 1$, or (iii) $z_{i}<0$ for some $i$, then return the 
violated constraint and exit. Then we check whether

$$
s \geq \max _{\boldsymbol{X} \in \mathcal{X}} \sum_{i \in N}\left(u_{i}\left(X_{i}\right) z_{i}+\sum_{j \neq i}\left(u_{i}\left(X_{i}\right)-u_{i}\left(X_{j}\right)\right) y_{i j}\right) \text {. }
$$

We rewrite the right hand side in (9) as

$$
\begin{aligned}
& \sum_{i \in N} u_{i}\left(X_{i}\right) z_{i}+\sum_{i, j \in N}\left(u_{i}\left(X_{i}\right)-u_{i}\left(X_{j}\right)\right) y_{i j} \\
& =\sum_{i \in N}\left(\left(z_{i}+\sum_{j \neq i} y_{i j}\right) u_{i}\left(X_{i}\right)-\sum_{j \neq i} y_{j i} u_{j}\left(X_{i}\right)\right) .
\end{aligned}
$$

We claim that the maximum in (9) is attained by an allocation $\boldsymbol{X}$ such that $X_{i} \in \mathcal{F}$ for all $i \in N$. To describe this, assume $X_{i} \notin \mathcal{F}$ for some $i$. There exists $X_{i}^{\prime} \subset X_{i}$ such that $X_{i}^{\prime} \in \mathcal{F}$ and $u_{i}\left(X_{i}^{\prime}\right)=u_{i}\left(X_{i}\right)$ by the definition of $u_{i}$. By the monotonicity of $u_{j}$, we have $u_{j}\left(X_{i}^{\prime}\right) \leq u_{j}\left(X_{i}\right)$ for $j \neq i$. These imply

$$
\begin{aligned}
& \left(z_{i}+\sum_{j \neq i} y_{i j}\right) u_{i}\left(X_{i}\right)-\sum_{j \neq i} y_{j i} u_{j}\left(X_{i}\right) \\
& \leq\left(z_{i}+\sum_{j \neq i} y_{i j}\right) u_{i}\left(X_{i}^{\prime}\right)-\sum_{j \neq i} y_{j i} u_{j}\left(X_{i}^{\prime}\right) .
\end{aligned}
$$

Therefore, we assume $X_{i} \in \mathcal{F}(\forall i \in N)$ in the following.

Since all the utility functions are matroid-additive, we further rewrite (10) as

$$
\begin{aligned}
& \sum_{i \in N}\left(\left(z_{i}+\sum_{j \neq i} y_{i j}\right) \sum_{e \in X_{i}} u_{i}(\{e\})-\sum_{j \neq i} y_{j i} \sum_{e \in X_{i}} u_{j}(\{e\})\right) \\
& =\sum_{i \in N} \sum_{e \in X_{i}}\left(\left(z_{i}+\sum_{j \neq i} y_{i j}\right) u_{i}(\{e\})-\sum_{j \neq i} y_{j i} u_{j}(\{e\})\right) . \\
& \text { Let } \gamma_{i}(e):=\left(z_{i}+\sum_{j \neq i} y_{i j}\right) u_{i}(\{e\})-\sum_{j \neq i} y_{j i} u_{j}(\{e\}) \\
& \text { for all } i \in N \text { and } e \in E \text {. Thus, we see that } \\
& \qquad \sum_{i \in N} u_{i}\left(X_{i}\right) z_{i}+\sum_{i, j \in N}\left(u_{i}\left(X_{i}\right)-u_{i}\left(X_{j}\right)\right) y_{i j}=\sum_{i \in N} \sum_{e \in X_{i}} \gamma_{i}(e) .
\end{aligned}
$$

Therefore, the maximization problem in (9) is equivalent to the following problem:

$$
\begin{aligned}
\max & \sum_{i \in N} \sum_{e \in X_{i}} \gamma_{i}(e) \\
\text { s.t. } & \boldsymbol{X} \in \mathcal{X}, \\
& X_{i} \in \mathcal{F} \quad(\forall i \in N) .
\end{aligned}
$$

This is exactly MaxUSW in which every utility function is a constrained-additive one with the matroid $\mathcal{F}$. This problem is solved in polynomial time by using Edmonds' matroid intersection algorithm (Edmonds 1970). Summarizing these arguments, we obtain Theorem 6.

\section{Conclusion}

In this paper, we addressed fair allocation of indivisible goods to agents in terms of max-min fairness. Specifically, we focused on maximizing ESW with and without the envyfree constraint. Without the envy-free constraint, we provided a black-box gap-preserving reduction from MaxESW to MaxUSW by using the ellipsoid algorithm with an approximate separation algorithm. With the envy-free constraint, we proved that the envy-free MaxESW is NP-hard even when every agent has an additive utility with a matroid constraint, and we proposed a polynomial-time algorithm for the case where the matroid constraint is common to all agents.

\section{Acknowledgments}

We would like to thank the AAAI anonymous reviewers for their suggestions and comments. The first author is supported by JSPS KAKENHI Grant Number JP16K16005. The second author is supported by JSPS KAKENHI Grant Number JP17K12646.

\section{References}

Asadpour, A., and Saberi, A. 2010. An approximation algorithm for max-min fair allocation of indivisible goods. SIAM Journal on Computing 39(7):2970-2989.

Bansal, N., and Sviridenko, M. 2006. The Santa Claus problem. In Proceedings of the 38th annual ACM symposium on Theory of Computing, 31-40.

Bertelsen, A. 2005. Substitutes valuations and $\mathbf{M}^{\natural}$-concavity. M.Sc. Thesis, The Hebrew University of Jerusalem.

Bezáková, I., and Dani, V. 2005. Allocating indivisible goods. SIGecom Exchanges 5(3):11-18.

Bilò, V.; Caragiannis, I.; Flammini, M.; Igarashi, A.; Monaco, G.; Peters, D.; Vinci, C.; and Zwicker, W. S. 2018. Almost EnvyFree Allocations with Connected Bundles. In Proceedings of 10th Innovations in Theoretical Computer Science Conference, 14:1$14: 21$.

Bouveret, S.; Cechlárová, K.; Elkind, E.; Igarashi, A.; and Peters, D. 2017. Fair division of a graph. In Proceedings of the 26th International Joint Conference on Artificial Intelligence, 135-141.

Brandt, F.; Conitzer, V.; Endriss, U.; Lang, J.; and Procaccia, A. D. 2016. Handbook of Computational Social Choice. Cambridge University Press.

Budish, E. 2011. The combinatorial assignment problem: Approximate competitive equilibrium from equal incomes. Journal of Political Economy 119(6):1061-1103.

Caragiannis, I.; Kurokawa, D.; Moulin, H.; Procaccia, A. D.; Shah, N.; and Wang, J. 2016. The unreasonable fairness of maximum nash welfare. In Proceedings of the 17th ACM Conference on Economics and Computation, 305-322. ACM.

Chakrabarty, D., and Goel, G. 2010. On the approximability of budgeted allocations and improved lower bounds for submodular welfare maximization and gap. SIAM Journal on Computing 39(6):2189-2211.

Edmonds, J. 1970. Submodular functions, matroids, and certain polyhedra. In Combinatorial Structures and Their Applications. Gordon and Breach, New York. 69-87.

Foley, D. 1967. Resource allocation and the public sector. Yale Economic Essays 7:45-98.

Garey, M. R., and Johnson, D. S. 1979. Computers and Intractability: A Guide to the Theory of NP-Completeness. Freeman New York.

Golovin, D. 2005. Max-min fair allocation of indivisible goods. Technical Report CMU-CS-05-144, Carnegie Mellon University. 
Grötschel, M.; Lovász, L.; and Schrijver, A. 2012. Geometric algorithms and combinatorial optimization, volume 2. Springer Science \& Business Media.

Haeupler, B.; Saha, B.; and Srinivasan, A. 2011. New constructive aspects of the Lovász local lemma. Journal of the ACM 58(6):28:128:28.

Jansen, K. 2003. Approximate strong separation with application in fractional graph coloring and preemptive scheduling. Theoretical Computer Science 302(1-3):239-256.

Kelso, Jr, A. S., and Crawford, V. P. 1982. Job matching, coalition formation, and gross substitutes. Econometrica 50:1483-1504.

Khachiyan, L. 1980. Polynomial algorithms in linear programming. USSR Computational Mathematics and Mathematical Physics 20(1):53-72.

Khot, S.; Lipton, R. J.; Markakis, E.; and Mehta, A. 2008. Inapproximability results for combinatorial auctions with submodular utility functions. Algorithmica 52(1):3-18.

Nisan, N.; Roughgarden, T.; Tardos, É.; and Vazirani, V. V. 2007. Algorithmic Game Theory. Cambridge University Press.

Oxley, J. G. 2011. Matroid Theory, 2nd Ed. Oxford University Press, USA.

Rothe, J. 2016. Economics and Computation. Springer.

Steinhaus, H. 1948. The problem of fair division. Econometrica 16(1):101-104.

Varian, H. R. 1974. Equity, envy, and efficiency. Journal of Economic Theory 9(1):63-91.

Vondrák, J. 2008. Optimal approximation for the submodular welfare problem in the value oracle model. In Proceedings of the fortieth annual ACM symposium on Theory of computing, 67-74. 